\title{
Range-extended EXAFS at the $L$ edge of rare earths using high-energy-resolution fluorescence detection: A study of La in LaOCl
}

\author{
P. Glatzel \\ European Synchrotron Radiation Facility, BP 220, F-38043 Grenoble Cedex, France \\ F. M. F. de Groot, O. Manoilova, D. Grandjean, and B. M. Weckhuysen \\ Department of Inorganic Chemistry and Catalysis, Debye Institute, Utrecht University, Sorbonnelaan 16, 3584 CA Utrecht, \\ The Netherlands \\ U. Bergmann \\ Stanford Synchrotron Radiation Laboratory, P.O. Box 20450 Stanford, California 94309, USA \\ R. Barrea \\ BioCAT, Advanced Photon Source, Argonne National Laboratory, 9700 S. Cass Av., Argonne, Illinois 60439, USA
}

(Received 7 February 2005; published 15 July 2005)

\begin{abstract}
We present extended x-ray absorption fine structure (EXAFS) data at the $\mathrm{La} L_{2}$ edge in $\mathrm{LaOCl}$ that were recorded on the $L \beta_{1}$ fluorescence line with an analyzer energy bandwidth of $1.3 \mathrm{eV}$. We show that by taking advantage of the high-energy-resolution fluorescence detection (HERFD) it is possible to extend the energy range for $L_{2}$ EXAFS analysis beyond the $L_{1}$ edge if the sample is optically thin. The arguments presented here generally apply to fluorescence-detected absorption spectroscopy if the fluorescence lines are sufficiently separated in energy. Calculations using an atomic multiplet model show that intensity due to $2 p 4 d$ multipleelectron excitations is reduced in HERFD spectra. The technique has the potential of considerably improving EXAFS analyses at low energies $(<10 \mathrm{keV})$ when absorption edges lie within a few hundred electron volts.

DOI: 10.1103/PhysRevB.72.014117

PACS number(s): 61.10.Ht, 87.64.Fb, 87.64.Gb
\end{abstract}

\section{INTRODUCTION}

The extended x-ray absorption fine structure (EXAFS) bears information on the local coordination of the X-ray absorbing atom. ${ }^{1}$ EXAFS arises from the scattering of a photoexcited electron wave with momentum $k=\sqrt{\hbar \omega-\left|E_{0}\right|}$ off the coordination shells around the central atom with threshold energy $E_{0}$. Quantitative information can be extracted by fitting the oscillations in the EXAFS fine structure function $\chi(k)$ using the EXAFS formula ${ }^{2}$

$$
\begin{aligned}
\chi(k)= & S_{0}^{2} \sum_{\text {shell } j} \frac{N_{j} F_{j}(k)}{k R_{j}^{2}} \sin \left[2 k R_{j}+\delta_{j}(k)\right] \exp \left(-\frac{2 R_{j}}{\lambda(k)}\right) \\
& \times \exp \left(-2 k^{2} \sigma_{j}^{2}\right),
\end{aligned}
$$

with the many-body amplitude reduction factor $S_{0}^{2}$, the number of neighbors $N_{j}$ in shell $j$, the backscattering amplitude $F_{j}$, the distance to the x-ray absorbing atom $R_{j}$, and a phase shift $\delta_{j}$. The oscillations are dampened by two exponential factors. One accounts for the mean-squared fluctuation in $R_{j}$ due to structural disorder and thermal effects and is described by the EXAFS Debye-Waller factor $\sigma_{j}^{2}$. The second exponential factor includes inelastic losses of the outgoing electron as well as the finite lifetime of the core-hole excited state. The two effects are lumped together in the effective mean free path $\lambda(k)$ that increases monotonically with increasing $k$ in the EXAFS range in accordance with the "universal" mean-free-path curve of an electron beam in matter.
The maximum number of free parameters that is available to fit an EXAFS spectrum depends on the range of electron momentum $\Delta k$ (Ref. 3):

$$
\text { number of free parameters }=\frac{2 \Delta k \Delta R}{\pi}+2 .
$$

The radius interval $\Delta R$ determines how many shells around the central atom are included in the EXAFS analysis and thus how many parameters need to be fitted. It is thus desirable to record a large range of electron momentum $\Delta k$ for an accurate fitting up to high-coordination shells. The available $k$ range is limited by the dampening of the oscillations due to structural disorder (EXAFS Debye-Waller factor) and by the finite mean free path of the scattered electron. The $k$ range can also be limited by additional absorption edges in the EXAFS range due to (a) another element present in the sample or (b) another core-hole state of the central atom. We deal in this paper with case (b) but many arguments presented here also apply to (a).

EXAFS in rare earths is measured at either the $L$ or $K$ edge. The energy broadening from the short $1 s$ core hole lifetime [e.g., La: $14.1 \mathrm{eV}$ (Ref. 4)] strongly dampens the EXAFS oscillations at the $K$ edge. Performing the measurements at low temperatures partly remedies this dampening by reducing the EXAFS Debye-Waller factor. However, temperature is not an adjustable parameter in many in situ applications-e.g., in catalysis. A deconvolution procedure was applied in other studies to remove the lifetime broadening and/or a large $k$ range was used at the $K$ edge ${ }^{5,6}$ But an 


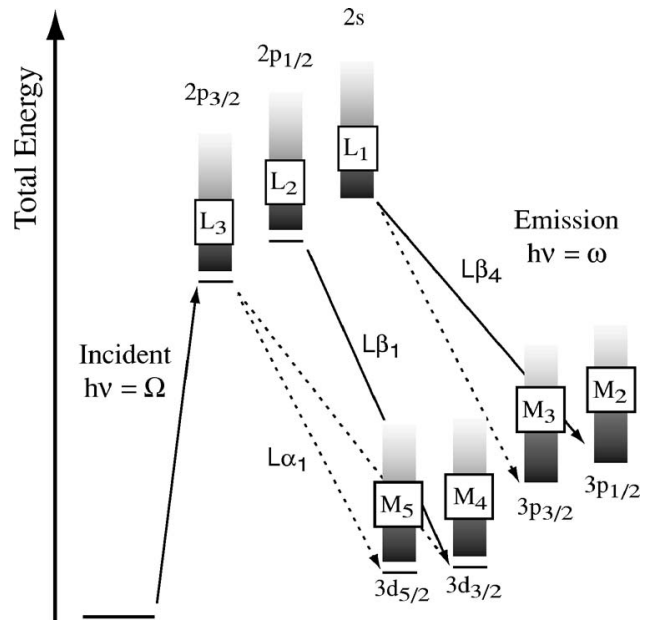

FIG. 1. Simplified energy scheme for La $L$ absorption and emission. The electron configurations are described using only the core hole with its spin-orbit splitting. The discrete resonances before the edges indicate the strong $2 p-5 d$ transitions.

EXAFS fitting beyond the first-coordination shell proved difficult with the sample kept above the Debye temperature. In particular, for dilute samples the data quality is poor at high $k$ (Ref. 7) and alternative techniques are desirable.

The $L$ edge has the advantage of a longer core-hole lifetime (La: $3.4 \mathrm{eV}$ ) but the EXAFS energy range at, e.g., the $L_{3}$ edge that can be used for an EXAFS analysis is limited by the $L_{2}$ edge (Fig. 1). Another difficulty arises from multipleelectron excitations, in particular $4 d$ to $5 d$ shake-up transitions that occur in the $L$-edge EXAFS and often hamper a detailed analysis. ${ }^{8,9}$

We present a technique to record EXAFS spectra at the $L$ edge of rare earths that (i) discriminates against unwanted edges in the EXAFS region and (ii) suppresses intensity due to multiple-electron excitations.

The technique is based on detection of x-ray $L$ fluorescence with a high-energy-resolution analyzer. Fluorescencedetected absorption spectroscopy is often carried out using energy-dispersive solid-state (e.g., Ge) detectors with an energy resolution $\Delta E / E$ of about 0.05 . It is possible to improve the resolution by a factor of $>100$ by using an $x$-ray analyzer based on perfect crystal Bragg optics. The small energy bandwidth yields distinct fluorescence lines for different decay channels. High-energy-resolution fluorescence detection (HERFD) with an energy bandwidth similar to the core-hole lifetime broadening is less efficient due to the loss in solid angle. This technique therefore requires an intense, tunable $\mathrm{x}$-ray source as available at third-generation synchrotron radiation facilities.

\section{EXPERIMENT}

We performed the experiments at the undulator beamline 18ID BioCAT of the Advanced Photon Source at Argonne National Laboratory. ${ }^{10}$ The incident energy was selected by means of a liquid-nitrogen-cooled $\mathrm{Si}(111)$ double-crystal monochromator. Higher harmonics were suppressed by a focusing mirror, and the beam size on the sample was $1 \mathrm{~mm}$
TABLE I. The $L$ and $M$ edges as well as $L M$ fluorescence decay channels of selected elements between silver $(Z=47)$ to ytterbium $(Z=70)$. The crucial energy range accessible for normal EXAFS are given in the bottom two rows.

\begin{tabular}{ccccccc}
\hline \hline & Ag 47 & Te 52 & La 57 & Pr 59 & Gd 64 & Yb 70 \\
\hline$L_{1}$ & 3806 & 4939 & 6266 & 6835 & 8375 & 10486 \\
$L_{2}$ & 3523 & 4612 & 5891 & 6440 & 7930 & 9978 \\
$L_{3}$ & 3351 & 4341 & 5483 & 5964 & 7242 & 8943 \\
$M_{1}$ & 719 & 1006 & 1362 & 1511 & 1881 & 2398 \\
$M_{2}$ & 604 & 871 & 1209 & 1337 & 1688 & 2173 \\
$M_{3}$ & 573 & 820 & 1128 & 1242 & 1544 & 1950 \\
$M_{4}$ & 374 & 583 & 853 & 948 & 1221 & 1576 \\
$M_{5}$ & 368 & 573 & 836 & 929 & 1190 & 1528 \\
$L_{1} M_{3}$ & 3233 & 4119 & 5138 & 5593 & 6831 & 8536 \\
$L_{1} M_{2}$ & 3202 & 4068 & 5057 & 5498 & 6687 & 8313 \\
$L_{2} M_{4}$ & 3149 & 4029 & 5038 & 5492 & 6709 & 8402 \\
$L_{3} M_{4}$ & 2977 & 3758 & 4630 & 5016 & 6021 & 7367 \\
$L_{3} M_{5}$ & 2778 & 3521 & 4355 & 4722 & 5698 & 6993 \\
$L_{1}-L_{2}$ & 283 & 327 & 375 & 395 & 445 & 508 \\
$L_{2}-L_{3}$ & 172 & 271 & 408 & 476 & 688 & 1035 \\
\hline \hline
\end{tabular}

horizontal by $1.4 \mathrm{~mm}$ vertical with a total flux on the order of $10^{13}$ photons/sec. We used an analyzer that employs the (331) Bragg plane of one spherically bent $(R=860 \mathrm{~mm}) \mathrm{Ge}$ wafer with $89 \mathrm{~mm}$ diameter. A $1.3 \mathrm{eV}$ energy bandwidth of the crystal analyzer was determined at $5040 \mathrm{eV}$ by measuring the elastic peak and assuming theoretical broadening of the $\mathrm{Si}(111)$ monochromator $(0.8 \mathrm{eV})$.

As a model system we chose $\mathrm{La}$ in polycrystalline $\mathrm{LaOCl}$, which is an important catalytic material and is actively studied for, e.g., the destruction of chlorinated hydrocarbons. ${ }^{11,12}$ The $\mathrm{LaOCl}$ sample was crushed to a fine powder and appropriately diluted with $\mathrm{BN}$.

The data reduction (pre- and post-edge fitting, atomic background subtraction) was performed using the IFEFFIT engine. ${ }^{13}$ The $R b k g$ value was set such that none of the lowfrequency peaks in the Fourier-transformed spectrum was suppressed. Below this value, the EXAFS oscillations in $k$ space in the present case are independent of the $R b k g$ value. EXAFS fitting was performed using EXCURVE98. ${ }^{14}$

\section{RESULTS}

Figure 1 shows a simplified energy scheme for the $L$ absorption edges and the strongest radiative decay channels to final-state configurations with a hole in the $M$ shell. The respective excitation energies, decay energies, and crucial energy differences are given for selected elements in Table I (Ref. [4]). The La electron configuration in the ionic limit $\left(\mathrm{La}^{3+}\right)$ for $\mathrm{LaOCl}$ is that of Xe with an empty $5 d$ shell. Experimental spectra for the La absorption $L$ edges recorded in transmission mode and the $L \beta_{1}$ and $L \beta_{4}$ emission lines are shown in Fig. 2. The emission lines, normalized to the spectral area, are shown for incident energies below and above the $L_{1}$ edge. The $L \beta_{4}$ line can be easily identified by com- 

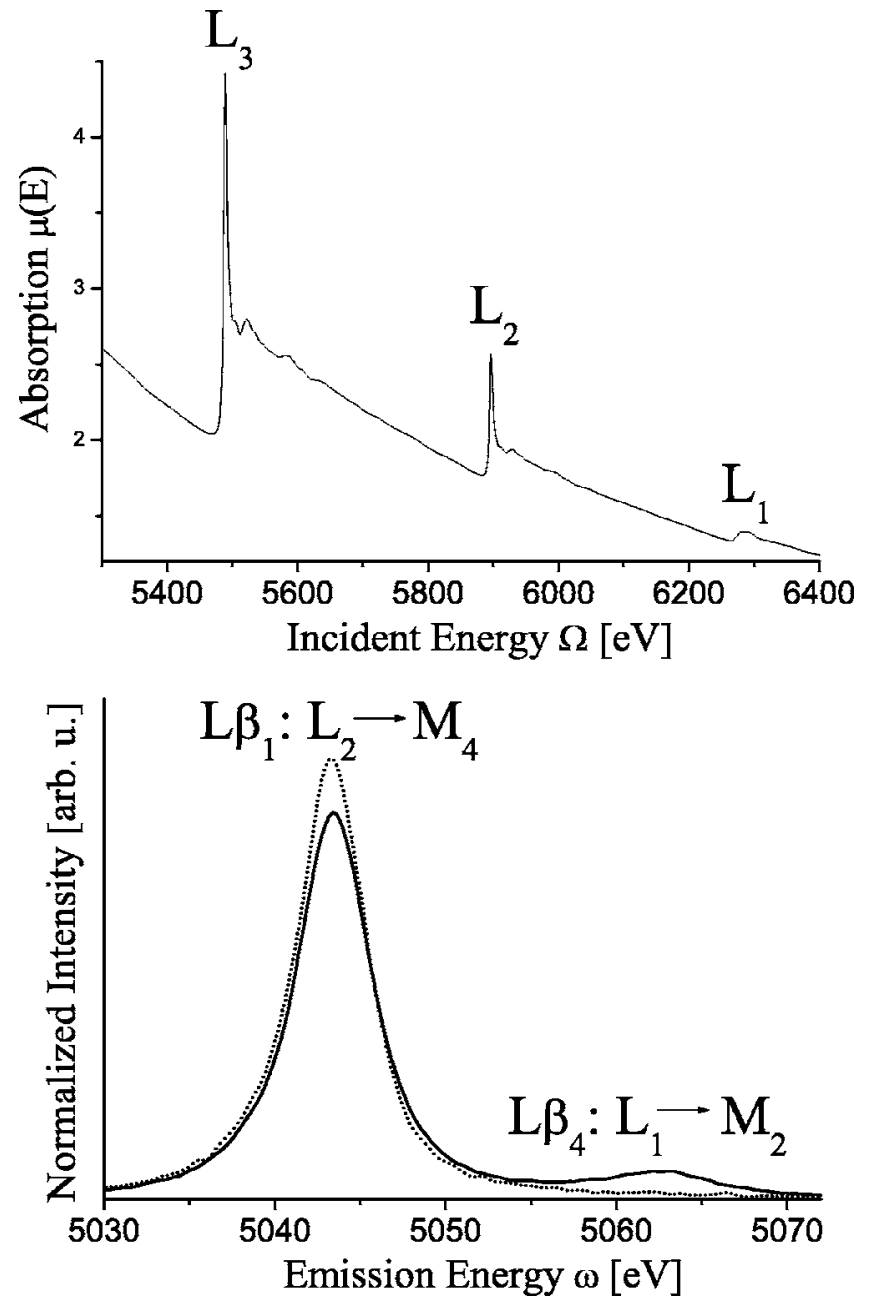

FIG. 2. Top: La absorption in $\mathrm{LaOCl}$ (transmission spectrum). Bottom: fluorescence (emission) spectra recorded above the $L_{3}$ edge at $6400 \mathrm{eV}$ incident energy (solid) and below at $6000 \mathrm{eV}$ (dotted).

paring the two spectra. HERFD-absorption-like spectra can be recorded by tuning the emission spectrometer to the maximum fluorescence intensity of the $L \beta_{1}$ emission line and scanning the incident energy through the $L_{2}$ absorption.

The $L \beta_{1}$ emission line intensity only depends on the probability of a $2 p_{1 / 2}\left(L_{2}\right)$ core-hole creation. It is therefore possible to record $L_{2}$ EXAFS spectra that extend beyond the $L_{1}$ edge with a large $k$ range. However, when the incident energy is tuned above the $L_{1}$ edge another interaction channel for the incoming photons becomes possible. As a result, the $L \beta_{1}$ line intensity decreases. The fluorescence intensity $I_{f}$ normalized to the incident X-ray intensity $I_{0}$ is related to the total absorption $\mu_{\text {tot }}$ of the sample (with thickness $d$ ) and the absorption of interest $\mu$ (Ref. 1):

$$
\begin{aligned}
& \frac{I_{f}}{I_{0}} \propto \frac{\mu(E)}{\frac{\mu_{t o t}(E)}{\sin \theta}+\frac{\mu_{t o t}\left(E_{f}\right)}{\sin \varphi}}\left\{1-\exp \left[-\left(\frac{\mu_{t o t}(E)}{\sin \theta}\right.\right.\right. \\
& \left.\left.\left.+\frac{\mu_{t o t}\left(E_{f}\right)}{\sin \varphi}\right) d\right]\right\} \text {. }
\end{aligned}
$$

The incident and fluorescence energies are denoted $E$ and $E_{f}$,

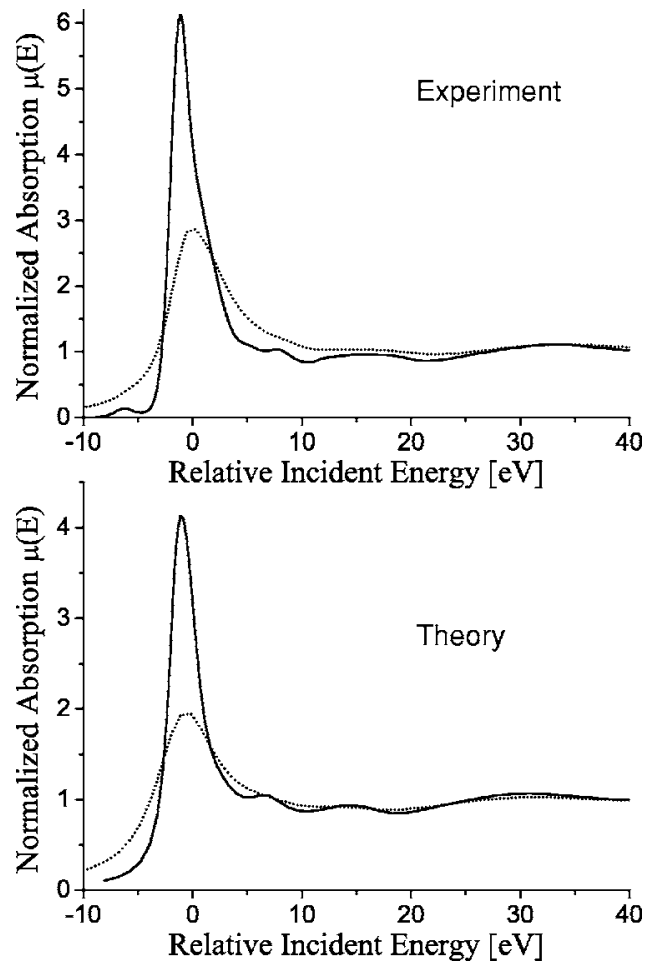

FIG. 3. Top: experimental La absorption spectra in transmission mode ( $L_{3}$ edge, dotted line) and using the HERFD technique $\left(L_{2}\right.$ edge, solid line). Bottom: calculated spectra taking the full (dotted line) and reduced (solid line) core-hole lifetime broadening into account.

respectively. The angles $\theta$ and $\phi$ describe the orientation of the sample surface to the incoming and outgoing $\mathrm{x}$-ray beams, respectively. For the present case, $\mu(E)=\mu_{L_{2}}^{L a}(E)$-i.e., the "clean" La $L_{2}$ absorption-and $\mu_{\text {tot }}(E)=\mu_{L_{2}}^{L a}(E)+\mu_{L_{1}}^{L a}(E)+\mu_{\text {other }}$ includes the absorption of all La edges as well as all other elements present in the sample. A HERFD absorption scan detected in the $L \beta_{1}$ emission line of a concentrated sample therefore exhibits a drop in intensity at the $L_{1}$ edge. By making the sample optically thin only the first-order expansion term of the exponential function contributes. The angular-dependent terms cancel in this case and the fluorescence signal $I_{f}$ becomes proportional to $\mu(E)$.

We note that this is the standard requirement for conventional fluorescence-detected absorption spectroscopy to avoid thickness effects. For the present case, the drop in $I_{f}$ due to the La $L_{1}$ edge can be reduced by diluting the sample until it is sufficiently smaller than the EXAFS oscillations. We note, furthermore, that the same argument applies to systems where the EXAFS range of interest is limited by the edge of another element in the sample.

We compare conventional (transmission) absorption spectra to the high-resolution spectra in Fig. 3. In case of the transmission spectrum, we show the $L_{3}$ edge because the $L_{3}$ EXAFS oscillations superimpose on the $L_{2}$ features. The slightly different lifetime broadenings between the $L_{3}$ and $L_{2}$ edges are negligible. The $L_{3}$ EXAFS oscillations are not present in the $L_{2}$ HERFD EXAFS following the same arguments as for the $L_{1}$ edge. 


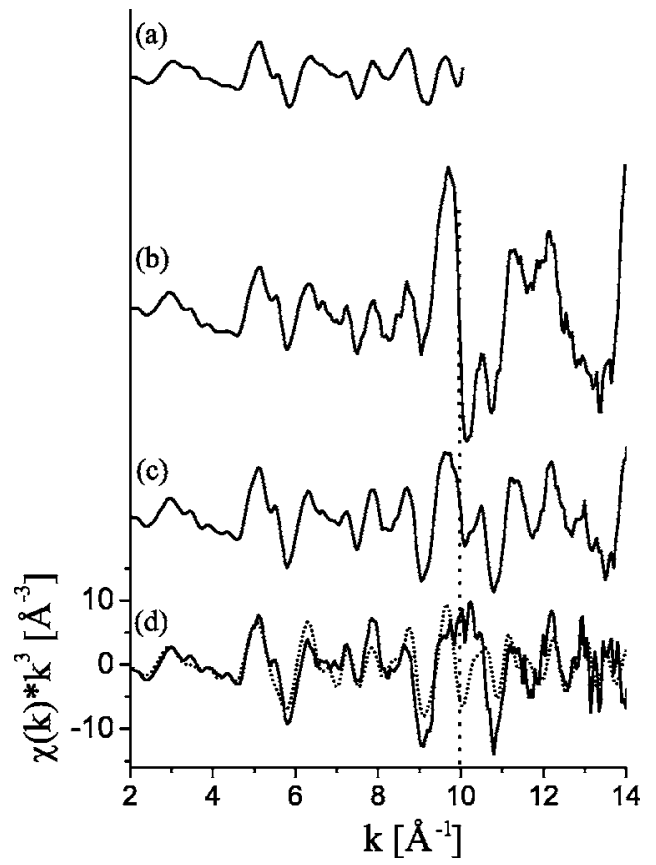

FIG. 4. La EXAFS in LaOCl: (a) $L_{3}$ transmission spectrum; $L_{2}$ $L \beta_{1}$ detected in (b) thick, concentrated sample, (c) diluted to $50 \%$, and (d) diluted to $10 \%$ of the $L \beta_{1}$ fluorescence signal compared to FEFF calculations (dotted). The position of the $L_{1}$ edge is indicated by a vertical line.

The sharpening of the $\mathrm{x}$-ray absorption near-edge spectroscopy (XANES) features has been reported before. ${ }^{15,16}$ The feature at about $-6 \mathrm{eV}$ relative incident energy arises from a $2 p$ to $4 f$ quadrupole transition that is only revealed in the high-resolution data. This feature was reported before by, e.g., Journal et al. at the $L_{3}$ edge of single-crystalline $\mathrm{LaF}_{3}$ and Dallera et al. in $\mathrm{Yb}$ systems. ${ }^{17,18}$

We performed calculations of the absorption fine structure with the FEFF $8.2 \operatorname{code}^{19}$ using the published $\mathrm{LaOCl}$ crystal structure. ${ }^{20,21}$ The space group is $P 4 / \mathrm{nmm}$, and the unit vectors were corrected using the results from powder diffraction data that were taken on the present sample. The radius for self-consistent-field (SCF) calculations was set to $4.2 \AA$. Full multiple-scattering (FMS) calculations were included for the XANES simulations with a radius of $5.25 \AA$. Increasing these values did not yield better results. By evaluating the calculated and experimental spectra for the $2 p$ to $5 d$ resonance we adjusted the $\mathrm{La} L_{2}$ core-hole lifetime broadening from the reported value of $3.7 \mathrm{eV}$ (Ref. 4) to $0.7 \mathrm{eV}$ for the HERFD spectra. Additionally, a $0.8 \mathrm{eV}$ broadening was applied to account for the instrumental energy bandwidth and a global $\sigma^{2}=0.006$ was used. The FEFF 8.2 calculations give a very good simulation of the experimental XANES region. The experimentally observed sharpening of the XANES features is reproduced. We were, however, not able to reproduce the $2 p$ to $4 f$ excitations below the main edge by including quadrupole transitions in the calculations.

In Figs. 4(b)-4(d), $L \beta_{1}$-detected EXAFS spectra are compared for different concentrations of $\mathrm{LaOCl}$ in $\mathrm{BN}$. Also shown is the $L_{3}$ transmission spectrum [Fig. 4(a)]. The intensity drop due to the $L_{1}$ edge at $k=10 \AA^{-1}$ disappears for

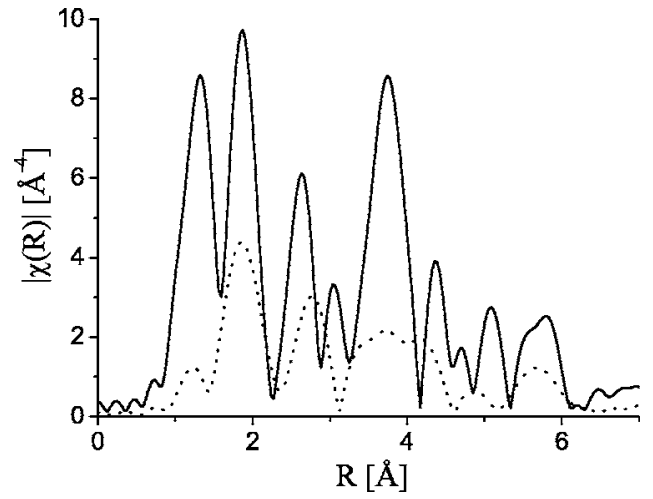

FIG. 5. Fourier transform of the $L_{3}$ transmission spectrum (dotted line, $k=2.0-10.0 \AA^{-1}$ ) and $L \beta_{1}$ HERFD spectrum (solid line, $\left.k=2.0-14.2 \AA^{-1}\right)$.

dilutions that give $10 \%$ of the fluorescence signal as compared to the concentrated sample. The La concentration in this sample is $0.9 \mathrm{wt} \%$. The total data acquisition time was $150 \mathrm{~min}$ for the most dilute sample with 10000 counts/sec in the EXAFS region. We note that HERFD absorption spectra are virtually background free. The samples for Figs. 4(b) and 4(c) also give saturated XANES features (not shown here).

The calculated EXAFS oscillations match the experimental spectrum rather well below and above the $L_{1}$ edge [Fig. 4(d)]. However, a discrepancy is observed at $k=10 \AA^{-1}$ which corresponds to the position of the $L_{1}$ edge. The intensity does not follow the oscillations but an additional decay channel appears to feed into the $L \beta_{1}$ fluorescence intensity. This point will be addressed in the discussion section.

We show a comparison of the Fourier transform for the conventional $L_{3}$ absorption data with the high-resolution $L \beta_{1}$-detected $L_{2}$ absorption spectrum in Fig. 5. The amplitudes, in particular of the peak due to La-La scattering, is greatly enhanced in the high-resolution spectra. Distinct peaks with amplitudes significantly above the background are visible in the HERFD data up to $4 \AA$. Using $\Delta R$ $=2.42 \AA(1.7-4.12 \AA)$ and $\Delta k=6 \AA^{-1}\left(4-10 \AA^{-1}\right)$ for the transmission and $\Delta k=10 \AA^{-1}\left(4-14 \AA^{-1}\right)$ for the HERFD spectrum in Eq. (1) we obtain 11 and 17 as the maximum number of independent parameters for the transmission and HERFD EXAFS analysis, respectively.

The HERFD EXAFS data were fitted between 1.7 and $4.12 \AA$ (Table II, Fig. 6). The coordination numbers were kept at fixed values corresponding to the tetragonal $P 4 / \mathrm{nmm}$ $\mathrm{LaOCl}$ structure that was clearly identified in the XRD studies. Distances, EXAFS Debye-Waller factors, and the threshold energy $E_{0}$ were the only parameters that were allowed to vary. The crystallographic distances are reproduced in the fit within $0.02 \AA$.

We now turn to multiple-electron excitations (MEE's) and argue that their intensity is suppressed in HERFD absorption spectroscopy. In experiments, the $2 p 4 d$ MEE's have been observed in rare earths, in particular at their $X$-MCD spectra. ${ }^{22}$ A simplified energy scheme is shown in Fig. 7. We denote the emission line arising from the $4 d$ to $5 d$ shake-up state $L N \beta_{1}$. An estimate using self-consistent field Hartree- 
TABLE II. Interatomic radial distances from the $\mathrm{LaOCl}$ crystal structure and EXAFS-fit results.

\begin{tabular}{cccc}
\hline \hline & & \multicolumn{2}{c}{ HERFD fit } \\
Shell & $\begin{array}{c}\text { Crystal structure } \\
R[\AA]\end{array}$ & $R[\AA]$ & $2 \sigma^{2}\left[\AA^{2}\right]$ \\
\hline $4 \mathrm{O}$ & 2.372 & 2.387 & 0.007 \\
$1 \mathrm{Cl}$ & 3.121 & 3.146 & 0.020 \\
$4 \mathrm{Cl}$ & 3.186 & 3.722 & 0.005 \\
$1 \mathrm{Cl}$ & 3.717 & 3.773 & 0.013 \\
$4 \mathrm{La}$ & 3.754 & 4.091 & 0.014 \\
$4 \mathrm{La}$ & 4.097 & & \\
\hline \hline
\end{tabular}

Fock calculations (Cowan's code ${ }^{23}$ ) suggests that the fluorescence energy (center of gravity) for $L N \beta_{1}$ transition is shifted by approximately $1 \mathrm{eV}$ relative to the $L \beta_{1}$ transition. Furthermore, the two-electron and spin-orbit interactions (multiplet structure) in the $2 p^{5} 4 d^{9} 5 d^{2}$ intermediate state change upon the decay into the $3 d^{9} 4 d^{9} 5 d^{2}$ final state, resulting in a large number of final states and shifted fluorescence energies.

We performed atomic multiplet calculations for $2 p 3 d$ resonant inelastic $\mathrm{x}$-ray scattering to illustrate this point and considered $4 d$ to $5 d$ shake-up transitions by including the configurations $5 d^{0}$ and $4 d^{9} 5 d^{1}$ in the ground, $2 p^{5} 5 d^{1}$ and $2 p^{5} 4 d^{9} 5 d^{2}$ in the intermediate, and $3 d^{9} 5 d^{1}$ and $3 d^{9} 4 d^{9} 5 d^{2}$ in the final state. The absolute energy $E\left(2 p^{5} 5 d^{1}\right)$ was taken
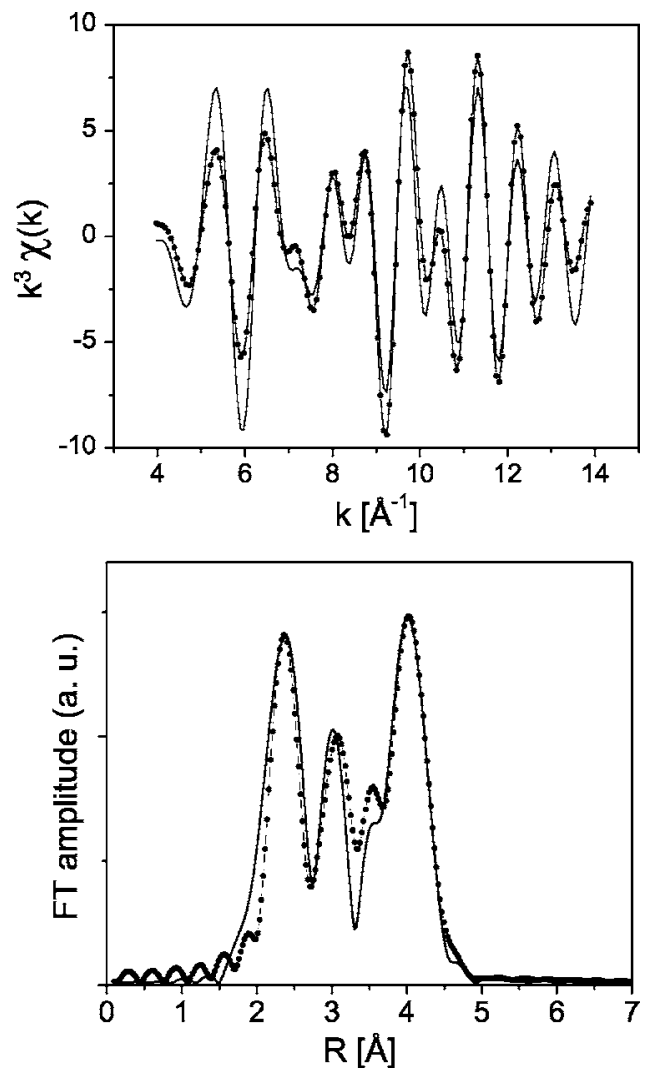

FIG. 6. Top: Fourier-filtered $(R=1.7-4.2 \AA)$ backward transform of the experimental range-extended EXAFS spectrum (dots) with fit (solid line). Bottom: Fourier transform.
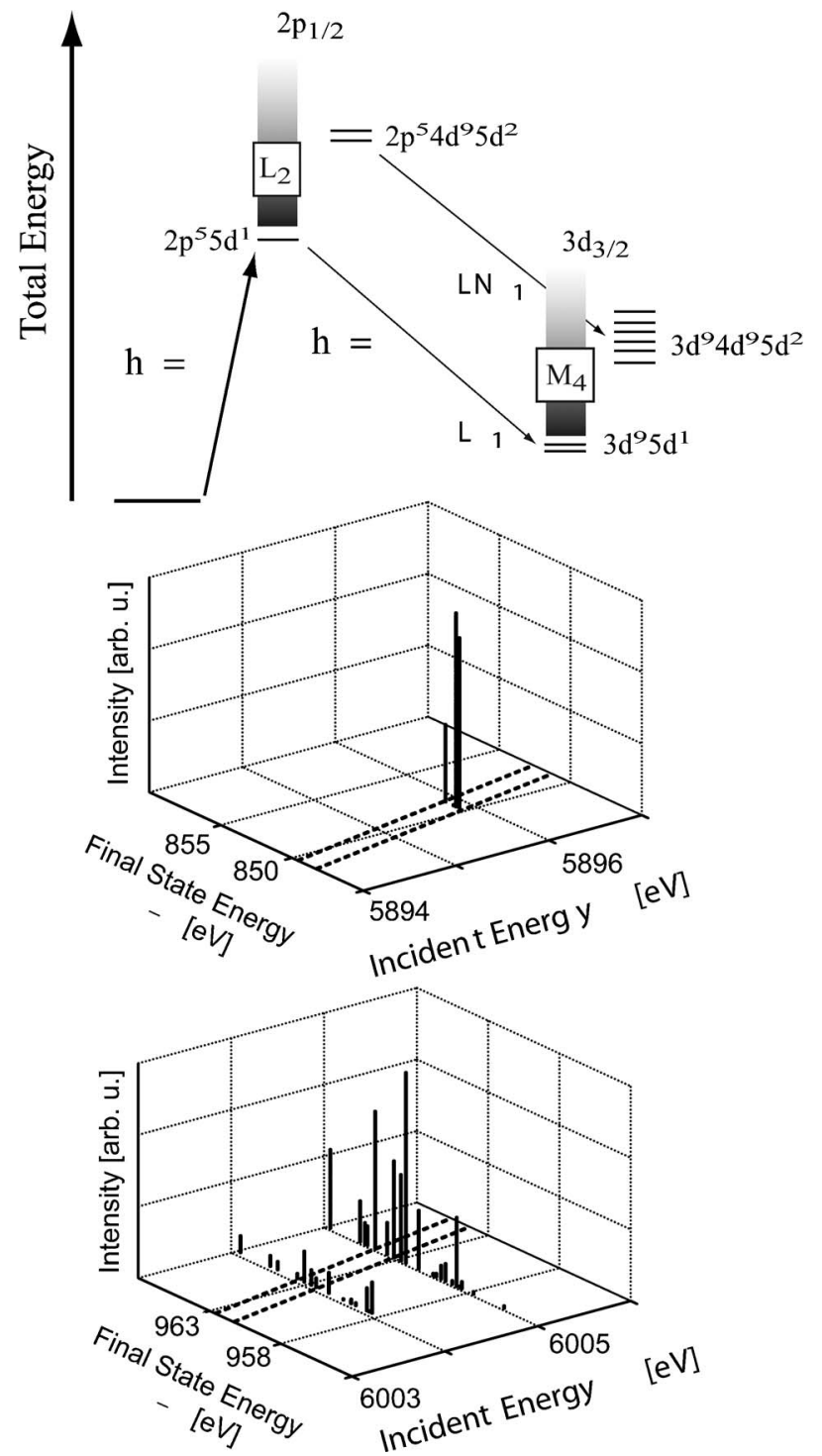

FIG. 7. Top: simplified energy scheme including $4 d$ to $5 d$ shake-up transitions. The splitting of the discrete resonances schematically indicates the multiplet splitting. Below: atomic multiplet calculations for $2 p_{1 / 2} 3 d_{3 / 2}$ resonant inelastic $\mathrm{x}$-ray scattering at the $L_{2}$ (center) and $L_{2} N_{4,5}$ (bottom) edges with a $4 d$ to $5 d$ shake-up transition. The dashed lines indicate the energy bandwidth [full width at half maximum (FWHM)] of the fluorescence analyzer (1.3 $\mathrm{eV})$.

from experiment and the energy differences between the oneelectron and the shake-up transition were taken from reported values. ${ }^{24} \mathrm{~A} Z+1$ model was invoked to account for the core hole and the aforementioned $1 \mathrm{eV}$ difference between $L \beta_{1}$ and $L N \beta_{1}$ was included. The mixing strength was set such that the shake-up resonance has $\sim 1 \%$ of the $L_{2} 2 p$ to $5 d$ transition intensity. The values of the Slater integrals were scaled down to $90 \%$ of their atomic values. ${ }^{25}$

The calculations in Fig. 7 show that the shake-up final states are spread over $\sim 10 \mathrm{eV}$ which is considerably larger than the energy window defined by the analyzer energy bandwidth. (We note that upon resonant excitation the line width of the emission lines representing the final states is not 
determined by the $2 p_{1 / 2}$ core hole lifetime but by the instrumental energy bandwidth and the $3 \mathrm{~d}$ core hole lifetime [ $0.6 \mathrm{eV}$ (Ref. [4])].) This considerable spread in the finalstate energy is mainly due to the electron-electron Coulomb interactions as expressed in the Slater integrals $F_{2,4}(3 d, 4 d)$ with values between 5 and $10 \mathrm{eV}$. The $4 d$ core hole that is created in the shake-up process thus strongly interacts with $3 d$ core hole in the final state of the fluorescence transition. This interaction is absent in the single-electron $L \beta_{1}$ transition. As a result, the intensity of the $4 d$ to $5 d$ shake-up structure is reduced in the HERFD absorption spectrum as compared to the transmission spectrum because in the latter the intensity of all shake-up states is included. Reexamining Fig. 4 and comparing the transmission spectrum (a) with the HERFD spectra (b)-(d) we do not find any evidence for multiple-electron excitations. This suggests that their intensity is too low or that they are too broad to be detected as a separate entity.

\section{DISCUSSION}

The EXAFS data for different concentrations of $\mathrm{LaOCl}$ in $\mathrm{BN}$ demonstrate that it is possible to suppress the influence of unwanted absorption cross sections [cf. Eq. (3)]. It is in general not important whether the unwanted edge arises from the same or a different element in the sample. The restriction of using optically thin samples generally applies to fluorescence detected absorption spectroscopy and is thus not characteristic for the new technique presented here. Since fluorescence detected absorption spectroscopy was developed in order to be able to measure EXAFS in dilute systems, HERFD EXAFS does not introduce additional constraints in terms of sample preparation.

However, a discontinuity occurs in the present case at $k$ $=10 \AA^{-1}$, which corresponds to the La $L_{1}$ edge, even for dilute samples. One possible explanation for this deviation from the EXAFS oscillations is that a radiative $2 s \rightarrow 2 p_{1 / 2}$ $\rightarrow 3 d_{3 / 2}$ cascade decay contributes to the $L \beta_{1}$ intensity above the $L_{1}$ edge (cf. Fig. 1). Another explanation could be that the $L \beta_{1}$ and $L \beta_{4}$ lines (cf. Fig. 2) possibly are not sufficiently separated in energy, giving $L_{1}$ contributions in the $L \beta_{1}$-detected EXAFS from the tail of the $L \beta_{4}$ emission line. A fit of the emission spectrum shows that about $0.01 \%$ intensity of the $L \beta_{4}$ line is still present in the maximum of the $L \beta_{1}$ line.

In future experiments this can be tested by using the $L \alpha_{1}$ $2 p_{3 / 2}$ to $3 d_{5 / 2}$ emission line that is sufficiently separated from all other emission lines. Furthermore, a radiative $2 p_{1 / 2}$ $\rightarrow 2 p_{3 / 2} \rightarrow 3 d_{3 / 2}$ cascade decay that would feed into the $L \alpha_{1}$ emission line intensity is electric dipole forbidden. A non radiative $2 p_{1 / 2} 2 p_{3 / 2}$ Coster-Kronig decay leads to doubly ionized states and thus different fluorescence energies.

The EXAFS oscillations above $k=10 \AA^{-1}$ nicely match the calculated data [cf. Fig. 4(d)]. While the discrepancy at $k=10 \AA^{-1}$ hampers a detailed EXAFS analysis, we still obtain the correct interatomic distances up to $4.1 \AA$. This presents already a notable improvement over conventional techniques, in particular for dilute systems that are difficult or impossible to measure in transmission at the $K$ edge.

The atomic multiplet calculations demonstrate the mechanism for suppression of intensity due to multielectron excitations. The importance of two-electron interactions (Slater integrals) in inner-shell spectroscopy is well documented, ${ }^{26,27}$ and the calculated and empirically scaled ${ }^{25}$ line splittings usually match the experimental spectra to better than $20 \%$. We thus expect an observable suppression of the shake-up transitions in the HERFD EXAFS. A more quantitative prediction for the reduction of shake-up intensity in the EXAFS requires extensive many-configuration calculations that are beyond the scope of the present study.

Comparing the transmission with high-resolution fluorescence detected spectrum [Figs. 4(a) and 4(d)] we do not find any evidence for multielectron excitations. This is confirmed by the FEFF calculations. The weak feature at $k \sim 5.4 \AA^{-1}$ in the experimental spectra (multiple-electron features have been observed at $k=5.6 \AA^{-1}$ ) also appears as a weak shoulder in the calculations. Furthermore, the intensity of this feature does not change between the transmission and HERFD spectrum.

We have thus introduced an additional tool to investigate multielectron excitations in absorption spectroscopy. The fact that we do not find shake up features in $\mathrm{LaOCl}$ even in the conventional transmission spectrum may be due the properties of the present system and does not question the existence of these features in other systems. Shake-up transitions have been observed, for example, in rare-earth-doped silica gels. ${ }^{9}$

Returning to the elements in Table I, we observe that throughout the rare-earth series the accessible energy ranges increase and that for $\mathrm{Yb}$, the $L_{3}$ range of over $1000 \mathrm{eV}$ is enough for good EXAFS spectra. Setting the limit at $700 \mathrm{eV}$, the heavy rare earths (beyond $\mathrm{Gd}$ ) are accessible with normal EXAFS at the $L_{3}$ edge. The early rare earths, La-Gd, would benefit most from range extended EXAFS.

In addition, we observe that short-edge energy ranges are found in particular for silver. Even shorter ranges occur for $\mathrm{Pd}$ and the earlier $4 d$ elements. These energy ranges are too short for any detailed EXAFS analysis, but range-extended EXAFS promises also for $4 d$ elements to allow EXAFS analysis of their $L$ edges. This would greatly enhance the importance of these edges, beyond their usage for XANES analysis.

Finally, the choice of the radiative decay channel ( $L M$ emission; cf. Table I) for fluorescence detection depends on the availability of suitable crystal analyzers as well as possible intermediate electronic transitions, as we might have observed in the present case, which could give rise to nonEXAFS features in the absorption spectra.

\section{SUMMARY AND CONCLUSIONS}

We have performed $L \beta_{1}$-detected high-energy-resolution $(1.3 \mathrm{eV})$ fluorescence-detected EXAFS at the $L_{2}$ edge of $\mathrm{La}$ for a dilute $\mathrm{LaOCl}$ sample beyond the onset of the $L_{1}$ edge. Our arguments with respect to the elimination of secondary edges in the EXAFS range generally apply to fluorescence detected absorption spectroscopy. We have furthermore 
shown within an atomic multiplet model that intensity due to shake-up transitions is suppressed using the high-energyresolution spectroscopy.

The technique can be applied to many systems with unwanted absorption edges in the EXAFS range of interest. The HERFD technique is, like conventional fluorescencedetected absorption spectroscopy, limited to optically thin samples that yield low count rates. The efficiency of the emission spectrometer can be increased considerably by enlarging the captured solid angle (multicrystal spectrometer). ${ }^{28}$ Depending on the energy separation between the fluorescence lines for different decay channels one can also increase the spectrometer efficiency by sacrificing energy resolution. Thus, various improvements are possible and high-energy-resolution fluorescence detection has the potential of becoming a standard tool that complements existing techniques of absorption spectroscopy.

\section{ACKNOWLEDGMENTS}

The authors thank the Netherlands Scientific Organisation-Chemical-Sciences (NWO-CW) for support. U.B. was supported by the Stanford Synchrotron Radiation Laboratory, Department of Energy, Office of Basic Energy Sciences Contract No. DE-AC03-765F00515. Use of the Advanced Photon Source was supported by the U.S. Department of Energy, Basic Energy Sciences, Office of Science, under Contract No. W-31-109-ENG-38. BioCAT is a National Institutes of Health-supported Research Center RR08630. We thank Stephen P. Cramer for insightful discussions.
${ }^{1} X$-ray Absorption: Principles, Applications, Techniques of EXAFS, SEXAFS, and XANES, edited by D. C. Koningsberger and R. Prins (Wiley, New York, 1988), Vol. 92.

${ }^{2}$ J. J. Rehr and R. C. Albers, Rev. Mod. Phys. 72, 621 (2000).

${ }^{3}$ E. A. Stern, Phys. Rev. B 48, 9825 (1993).

${ }^{4}$ Unoccupied Electronic States, edited by J. C. Fuggle and J. E. Inglesfield (Springer-Verlag, Berlin, 1992), Vol. 69.

${ }^{5}$ J. M. Cole, R. J. Newport, D. T. Bowron, R. F. Pettifer, G. Mountjoy, T. Brennan, and G. A. Saunders, J. Phys.: Condens. Matter 13, 6659 (2001).

${ }^{6}$ M. Borowski, D. T. Bowron, and S. De Panfilis, J. Synchrotron Radiat. 6, 179 (1999).

${ }^{7}$ T. Yamamoto, T. Tanaka, T. Matsuyama, T. Funabiki and S. Yoshida, J. Synchrotron Radiat. 8, 634 (2001).

${ }^{8}$ A. Kodre, I. Arcon, M. Hribar, M. Stuhec, F. Villain, W. Drube, and L. Tröger, Physica B 209, 379 (1995).

${ }^{9}$ J. Chaboy, A. Marcelli, and T. A. Tyson, Phys. Rev. B 49, 11652 (1994).

${ }^{10}$ R. A. Barrea, R. Fischetti, S. Stepanov, G. Rosenbaum, E. Kondrashkina, G. B. Bunker, E. Black, K. Zhang, D. Gore, R. Heurich, M. Vukonich, C. Karanfil, A. J. Kropf, G. Wang, and T. C. Irving, Phys. Scr. T115, 867 (2005).

${ }^{11}$ C. T. Au, H. He, S. Y. Lai, and C. F. Ng, Appl. Catal., A 159, 133 (1997).

${ }^{12}$ P. Van der Avert and B. M. Weckhuysen, Angew. Chem., Int. Ed. 41, 4730 (2002).

${ }^{13}$ M. Newville, P. Livins, Y. Yacoby, J. J. Rehr, and E. A. Stern, Phys. Rev. B 47, 14126 (1993).

${ }^{14}$ N. Binsted, J. W. Campbell, S. J. Gurman, and P. C. Stephenson, "SERC Daresbury Laboratory EXCURVE98 program." (1991) Details on obtaining the latest version of this code can be obtained from n.binsted@dl.ac.uk.
${ }^{15}$ F. M. F. de Groot, M. H. Krisch, and J. Vogel, Phys. Rev. B 66, 195112 (2002).

${ }^{16}$ K. Hämäläinen, D. P. Siddons, J. B. Hastings, and L. E. Berman, Phys. Rev. Lett. 67, 2850 (1991).

${ }^{17}$ L. Journel, J. M. Mariot, J. P. Rueff, C. F. Hague, G. Krill, M. Nakazawa, A. Kotani, A. Rogalev, F. Wilhelm, J. P. Kappler, and G. Schmerber, Phys. Rev. B 66, 045106 (2002).

${ }^{18}$ C. Dallera, M. Grioni, A. Shukla, G. Vanko, J. L. Sarrao, J. P. Rueff, and D. L. Cox, Phys. Rev. Lett. 88, 196403 (2002).

${ }^{19}$ A. L. Ankudinov, C. E. Bouldin, J. J. Rehr, J. Sims, and H. Hung, Phys. Rev. B 65, 104107 (2002).

${ }^{20}$ D. A. Fletcher, R. F. McMeeking and D. Parkin, J. Chem. Inf. Comput. Sci. 36, 746 (1996).

${ }^{21}$ L. H. Brixner and E. P. Moore, Acta Crystallogr., Sect. C: Cryst. Struct. Commun. 39, 1316 (1983).

${ }^{22}$ E. Dartyge, A. Fontaine, C. Giorgetti, S. Pizzini, F. Baudelet, G. Krill, C. Brouder, and J. P. Kappler, Phys. Rev. B 46, R3155 (1992).

${ }^{23}$ R. D. Cowan, The Theory of Atomic Structure and Spectra (University of California Press, Berkeley, 1981).

${ }^{24}$ G. P. Williams, in X-Ray Data Booklet, edited by A. C. Thompson and D. Vaughan (Lawrence Berkeley National Laboratory, Berkeley, 2001), p. 1.

${ }^{25}$ A. von dem Borne, R. L. Johnson, B. Sonntag, M. Talkenberg, A. Verweyen, P. Wernet, J. Schulz, K. Tiedtke, C. Gerth, B. Obst, P. Zimmermann, and J. E. Hansen, Phys. Rev. A 62, 052703 (2000).

${ }^{26}$ A. Kotani, J. Electron Spectrosc. Relat. Phenom. 100, 75 (1999).

${ }^{27}$ P. Glatzel and U. Bergmann, Coord. Chem. Rev. 249, 65 (2005).

${ }^{28}$ U. Bergmann and S. P. Cramer, in SPIE-The International Society for Optical Engineering (Society of Photo-Optical Instrumentation Engineers, San Diego, 1998), Vol. 3448, p. 198. 